\title{
Erratum to: Performance of a Structure Consisting a 2-Out-of-3:F Substructure Under Human Failure
}

\author{
Mangey Ram • Amit Kumar
}

Published online: 9 January 2015

(c) King Fahd University of Petroleum and Minerals 2014

Erratum to: Arab J Sci Eng (2014) 39:8383-8394

DOI 10.1007/s13369-014-1347-5

In Eq. (42) of the published paper, the $H_{1}, H_{2}, H_{3}, H_{4}, H_{5}$ equal to

$$
\begin{aligned}
& H_{1}=\frac{\lambda_{1}\left[\mu_{1}+\frac{\left(\lambda_{2}+\lambda_{3}\right) \mu}{(s+\mu)}+\frac{\lambda_{h} \mu_{h}}{\left(s+\mu_{h}\right)}+\frac{\lambda_{B} \mu_{A B}}{\left(s+\mu_{A B}\right)}\right]}{\left(s+\lambda_{B}+\lambda_{2}+\lambda_{3}+\lambda_{h}+\mu_{1}\right)} \\
& H_{2}=\frac{\lambda_{2}\left[\mu_{2}+\frac{\left(\lambda_{1}+\lambda_{3}\right) \mu}{(s+\mu)}+\frac{\lambda_{h} \mu_{h}}{\left(s+\mu_{h}\right)}+\frac{\lambda_{B} \mu_{A B}}{\left(s+\mu_{A B}\right)}\right]}{\left(s+\lambda_{B}+\lambda_{1}+\lambda_{3}+\lambda_{h}+\mu_{2}\right)} \\
& H_{3}=\frac{\lambda_{3}\left[\mu_{3}+\frac{\left(\lambda_{1}+\lambda_{2}\right) \mu}{(s+\mu)}+\frac{\lambda_{h} \mu_{h}}{\left(s+\mu_{h}\right)}+\frac{\lambda_{B} \mu_{A B}}{\left(s+\mu_{A B}\right)}\right]}{\left(s+\lambda_{B}+\lambda_{1}+\lambda_{2}+\lambda_{h}+\mu_{3}\right)} \\
& H_{4}=\left[\frac{\lambda_{h} \mu_{h}}{\left(s+\mu_{h}\right)}+\frac{\lambda_{B} \mu_{B}}{\left(s+\mu_{B}\right)}\right] \\
& H_{5}=\left(s+\lambda_{1}+\lambda_{2}+\lambda_{3}+\lambda_{B}+\lambda_{h}\right)
\end{aligned}
$$

The online version of the original article can be found under doi:10.1007/s13369-014-1347-5.

M. Ram ( $\varangle)$. A. Kumar

Department of Mathematics, Graphic Era University Dehradun, Uttarakhand, India

e-mail:drmrswami@yahoo.com
A. Kumar
e-mail: amit303singh@gmail.com 